\title{
Processo formativo do enfermeiro frente ao gerenciamento de resíduos de serviços de saúde: uma percepção discente
}

\author{
Nurse's formative process in the face of waste management of health services: a student perception \\ Proceso formativo de la enfermera frente a la gestión de residuos de los servicios de salud: una \\ percepción de los estudiantes
}

Recebido: 22/01/2021 | Revisado: 31/01/2021 | Aceito: 01/02/2021 | Publicado: 09/02/2021

\begin{abstract}
Resumo
Conhecer a percepção dos discentes de Enfermagem sobre Gerenciamento de Resíduos de Serviços de Saúde (GRSS). Estudo de abordagem qualitativa descritiva, desenvolvido em uma universidade pública do nordeste brasileiro. Utilizouse da técnica de Grupo Focal (GF) com sete discentes do quarto período do curso de graduação em Enfermagem. Reconheceram o enfermeiro como responsável pelo Plano de Gerenciamento de Resíduos de Serviços de Saúde (PGRSS) e destacaram a Educação Permanente em Saúde (EPS) como uma estratégia para capacitar a equipe sobre a temática, o que implica olhar além das práticas de saúde. O estudo constatou que existem fragilidades no processo de formação dos enfermeiros frente à temática RSS. Possivelmente, esse fato esteja associado à fase do curso vivenciada pelos discentes pesquisados. Destarte, esses resultados merecem ser investigados em estudos futuros, para aprofundamento, buscando soluções para melhorar o ensino sobre o tema.
\end{abstract}

Palavras-chave: Resíduos de serviços de saúde; Conhecimento; Estudantes de enfermagem.

\begin{abstract}
To know the perception of nursing students about Health Service Waste Management (GRSS). Descriptive qualitative study, developed in a public university in northeastern Brazil. The Focus Group (GF) technique was used with seven students from the fourth period of the undergraduate nursing course. They recognized the nurse as responsible for the Health Services Waste Management Plan (PGRSS) and highlighted Permanent Health Education (EPS) as a strategy to empower the team on the theme, which implies looking beyond health practices. The study found that there are weaknesses in the nursing education process in the face of the RSS theme. Possibly, this fact is associated with the phase of the course experienced by the students surveyed. Thus, these results deserve to be investigated in future studies, to deepen, seeking solutions to improve teaching on the subject.
\end{abstract}

Keywords: Waste of health services; Knowledge; Nursing students.

\section{Resumen}

Conocer la percepción de los estudiantes de enfermería sobre la Gestión de Residuos del Servicio de Salud (GRSS). Estudio cualitativo descriptivo, desarrollado en una universidad pública en el noreste de Brasil. La técnica del Grupo de Enfoque (GF) se utilizó con siete estudiantes del cuarto período del curso de enfermería de pregrado. Reconocieron a la enfermera como responsable del Plan de Gestión de Residuos de Servicios de Salud (PGRSS) y destacaron la Educación Permanente para la Salud (EPS) como una estrategia para capacitar al equipo en el tema, lo que implica mirar más allá de las prácticas de salud. El estudio encontró que hay debilidades en el proceso de educación de enfermería frente al tema RSS. Posiblemente, este hecho se asocia con la fase del curso experimentada por los estudiantes encuestados. Por lo tanto, estos resultados merecen ser investigados en futuros estudios, profundizar, buscando soluciones para mejorar la enseñanza sobre el tema.

Palabras clave: Residuos de servicios de salud; Conocimientos; Estudiantes de enfermería. 


\section{Introdução}

A sociedade moderna enfrenta sérios desafios, entre os quais, a complexidade e a diversidade existentes nos Resíduos Sólidos Urbanos (RSU) resultantes do processo histórico do qual emergem a Ciência Moderna e a Revolução Industrial (Moreschi, Rempel \& Backes, 2014). Entre os diversos tipos de RSU que resultam das ações antrópicas, os Resíduos de Serviços de Saúde (RRS), dentro de uma dimensão maior, constituem um desafio com interfaces, uma vez que além da questão ambiental inerente a qualquer resíduo, apresentam risco para a saúde do trabalhador e para a saúde pública (André, Veiga \& Takayanagui, 2016; Zarpelão, et al., 2016).

O Gerenciamento dos Resíduos de Serviços de Saúde (GRSS) adequado e seguro, de acordo com a Agência Nacional de Vigilância Sanitária (ANVISA), é um processo articulado e contínuo que depende de atualizações científicas e técnicas, regramento por instrumentos leais e normativos, além da qualificação dos recursos humanos (Brasil, 2018). Em concordância, o GRSS envolve planejamento, implantação e monitoramento das ações que objetivam evitar exposição, garantir a segurança dos usuários da saúde e profissionais envolvidos, como também prevenir a ocorrência de impactos ambientais, portanto, é uma questão de biossegurança (Uehara, Veiga \& Takayanagui, 2019).

Nessa realidade, os enfermeiros são responsabilizados pelo Plano de Gerenciamento de Resíduos de Serviços de Saúde (PGRSS) nos estabelecimentos de saúde, o que exige liderança, administração e gerenciamento de competências e habilidades inerentes ao processo gerencial do trabalho do enfermeiro (Cofen, 2005). Nesse contexto, as Diretrizes Curriculares Nacionais da Enfermagem (DCN/ENF) orientam o perfil do formando egresso com formação generalista capaz de atuar nos problemas/situações de saúde-doença, com senso de responsabilidade social e compromisso com a cidadania, como promotor integral da saúde do ser humano (Brasil, 2001). O referido documento, além de outros pressupostos, descreve que o enfermeiro deve estar apto a fazer o gerenciamento e a administração tanto da força de trabalho quanto de recursos físicos, materiais e de informação.

Moreschi et al. (2014) e Sanchez et al. (2018) advertem que o GRSS nem sempre é incorporado nos conteúdos teóricos e nas atividades práticas das Instituições de Ensino Superior (IES), podendo este profissional não reservar tempo para aprender a gerenciar os resíduos, o que dificulta a consolidação de novos conceitos e proatividade diante da problemática. Por consequência, os alunos, muitas vezes, acabam desconhecendo o PGRSS dos cursos nos quais estão inseridos e nos cenários de práticas (Gomes et al., 2014; Garbin et al., 2015).

A necessidade de maiores contribuições para a questão bem como o interesse como preceptora em contribuir com o aprimoramento do curso pesquisado, justificaram o desenvolvimento deste estudo, que teve como pergunta de investigação: $\mathrm{Na}$ perspectiva dos discentes, como o tema Resíduo de Serviços de Saúde vem sendo abordado na graduação? Na intenção de responder a essa questão, o estudo teve como objetivo conhecer a percepção dos discentes de Enfermagem sobre o gerenciamento dos RSS em uma universidade pública federal do nordeste brasileiro.

\section{Metodologia}

Estudo de abordagem qualitativa descritiva, desenvolvido em uma universidade pública do nordeste brasileiro, aprovado pelo Comitê de Ética em Pesquisa (CEP) da Universidade Federal de Alagoas (UFAL), conforme Parecer no 3.099.358. Para Pereira et. al (2018) para a utilização do método de pesquisa qualitativo é importante que haja interpretação por parte do pesquisador com suas opiniões sobre o fenômeno em estudo. Neles a coleta de dados muitas vezes ocorre por meio de entrevistas com questões abertas. O cenário de investigação foi o curso de graduação em Enfermagem de uma universidade pública, localizada no nordeste brasileiro. 
Na busca dos discentes de Enfermagem, inicialmente, foi efetuado contato com os representantes de turma dos $4^{\circ}, 5^{\circ}$, $6^{\circ}, 7^{\circ}$ e $8^{\circ}$ períodos do curso estudado. Em seguida, foi enviado um convite por e-mail para as respectivas turmas, além da divulgação do convite da pesquisa no mural do respectivo curso. Neste recorte do estudo, participaram sete discentes do $4^{\circ}$ período, sendo: cinco mulheres e dois homens. A faixa etária foi de 19 a 21 anos, e nenhum dos participantes havia realizado qualquer atividade complementar que abordasse a temática RSS. Para alcançar o objetivo desta pesquisa, utilizou-se a técnica de técnica de Grupo Focal (GF) com os discentes de Enfermagem para a produção das informações.

Após informar a todos sobre a gravação das discussões com o auxílio de dois dispositivos de telefone móvel (smartphone), reforçou-se a necessidade de respeito e o anonimato entre eles. Foram fornecidos esclarecimentos referentes ao Termo de Consentimento Livre e Esclarecido (TCLE). O documento foi lido e entregue a cada participante para ciência e assinatura. A transcrição do áudio foi realizada na íntegra e os participantes foram identificados com a letra "D" de discentes seguido de um numeral conforme a sequência inicial de participação no grupo. Os cenários de prática e as disciplinas citadas nas discussões foram identificados com as letras do alfabeto para evitar qualquer possibilidade de identificação. Os dados produzidos com base no GF foram armazenados, transcritos, sistematizados, categorizados e analisados.

\section{Resultados e Discussão}

Partindo destes pressupostos e baseado nas impressões pessoais, valores e opiniões, explicitados nas entrevistas, o processo de análise possibilitou a identificação da categoria temática empírica e suas subcategorias.

\section{Categoria: O processo de formação do enfermeiro sobre a temática GRSS}

Subcategoria: Oportunidades de aprendizagem no contexto teórico

Os dados coletados permitiram evidenciar que não existe uma disciplina específica no curso que aborde o tema GRSS. Pelos relatos, a abordagem do conteúdo é realizada de forma pontual e superficial. Os discentes narraram maior necessidade de debates e valorização do tema, de modo a favorecer uma mudança de comportamento dos sujeitos, como mostram os relatos:

\footnotetext{
"Disciplina não tem [...] a gente tem uma disciplina no curso, com uma aula só, que fala sobre resíduos, mas não é assim gerenciamento de resíduos" (D6).
}

"É necessário que esse conteúdo seja mais discutido durante o curso pra que a gente, como futuros enfermeiros, aprenda a respeitar melhor" (D7).

"Falando pelo que eu já passei na disciplina A teve um pouquinho, mas tipo não foi muito voltado para gerenciamento de resíduo em si, mas foi uns tópicos da aula da disciplina B, ela falou suscintamente, bem superficialmente" (D2).

Em um estudo que objetivou verificar o conhecimento sobre o descarte de resíduos infectantes e a sua prática entre acadêmicos dos cursos de Enfermagem e de Medicina de uma universidade, os resultados evidenciaram que existem falhas de formação na graduação em Saúde de forma que não privilegiem o pensamento crítico dessa temática e também não invistam adequadamente em pesquisas com esse enfoque (Souza et al., 2015).

Corroborando, outro estudo constatou a ausência ou fragmentação de abordagem sobre RSS, evidenciando que esta temática é pouco trabalhada na formação acadêmica dos cursos de graduação da área da Saúde (Moreschi et al. 2014; Oliveira et al. 2018), bem como na comunidade acadêmica, como um todo (Gomes et al., 2017). 
Nesse sentido, a interdisciplinaridade enfatizada por Moreschi et al. (2014) faz-se essencial para a compreensão e o domínio das etapas que envolvem o manejo dos RSS, bem como, para o complexo conhecimento de seu gerenciamento. E, como estratégias para vencer estes desafios, técnicas de ensino tais como a aprendizagem baseada em problemas e a problematização têm sido bem avaliadas pelos egressos de Enfermagem (Santos et al., 2019).

\section{Subcategoria: Oportunidades de aprendizagem no contexto prático}

Os depoimentos dos discentes do quarto período mostraram que as oportunidades de aprendizagem no contexto prático, até o momento do curso, ocorrem mediante vivências no laboratório de enfermagem, oportunidade extracurricular e, nos serviços de saúde, onde participam ativamente do processo de trabalho. As falas evidenciam que essas vivências aconteceram, em sua maioria, no ambiente hospitalar e parece não ocorrer de maneira previamente planejada ou programada.

\footnotetext{
"A gente viu na prática do laboratório e no serviço de saúde A técnica de calçar luva estéril que deve ter cuidado para não tocar em nada e saber tirar sem se contaminar" (D2).
}

Durante o GF, identificou-se, no laboratório de enfermagem e no estágio extracurricular, a ênfase em medidas de proteção individual e coletiva. Este resultado corrobora os desfechos da categoria que aborda os saberes sobre biossegurança e RSS. Para os discentes, o GRSS está atrelado à biossegurança quando traz à tona a segurança da comunidade hospitalar, a obrigatoriedade do uso de Equipamentos de Proteção Individual (EPIs) no manuseio dos RSS, como também a higienização das mãos.

O laboratório de enfermagem se constitui um espaço estratégico destinado ao desenvolvimento das práticas de ensino, visando às competências e habilidades do estudante, atuando no suporte ao processo de ensino-aprendizagem por professores e monitores.

Diversas pesquisas revelam a escassez de estudos envolvendo cenários de simulação e o manejo dos RSS. Nessa sequência, Eduardo et al. (2016) validaram o conteúdo de um cenário para ser empregado em simulação dos resíduos de saúde e verificaram sua exequibilidade, considerando um ambiente apto para atender ao objetivo da aprendizagem.

Em sequência, as atividades extracurriculares são definidas como ações desenvolvidas para além da realidade dos currículos, que ultrapassam a linha preestabelecida do curso. Essas atividades são configuradas como "currículo paralelo", desenvolvidas, simultaneamente, ao currículo oficial (Peres, Andrade \& Garcia, 2007) e foram lembradas pelos participantes da pesquisa, como espaço de prática.

\footnotetext{
"Não sei se tem relação, mas a técnica para retirar o jaleco, não aprendi aqui, ele deve ser retirado com cuidado, deixar pelo avesso num saco plástico. Aqui a gente compra uma bolsinha para guardar, mas nunca detalharam a técnica de tirar nem a limpeza da bolsa” (D6).
}

Nos ambientes de prática, os discentes relataram situações cotidianas em que as regras de biossegurança foram ignoradas. Ademais, também o erro cometido foi reconhecido pelo profissional:

\footnotetext{
"A gente viu em alguns setores do serviço de saúde A profissionais reencapando agulhas, realizando procedimentos sem luvas como também a caixa de descarpack cheia” (D8).
} 
"No serviço de saúde B às vezes a gente vai acompanhada por uma técnica de enfermagem, quando a professora não vai, daí uma vez eu fui e ela usava só um saco de lixo para os dois lixos comuns e contaminados, daí eu perguntei a ela porque só usava um, ela disse que sabia que estava errado, não falei nada, mas acho que ela estava economizando"(D9).

A falta de compromisso de formadores dos futuros profissionais, em relação ao manuseio do RSS, e a ausência de um modelo a ser imitado pelo aluno dificulta o desenvolvimento de adequada formação profissionais, refletindo em uma prática inapropriada (Corrêa, Lunardi \& Conto, 2007; Bento et al., 2017).

Percebeu-se, também, que existem fatores potencializadores de aprendizagem sobre RSS. Nos exemplos, os sujeitos conseguiram fazer a distinção entre os resíduos infectante, comum reciclável e não reciclável, nos cenários de prática, bem como reconheceram a necessidade de segregá-los na fonte. Estes saberes também foram mencionados durante a abordagem sobre os conceitos de GRSS.

\begin{abstract}
"Um descarte especifico agora no estágio a gente viu, perto da pia fica uma lixeira preta para jogar papel molhado e alimento e outra verde pra jogar papel seco" (D5).
\end{abstract}

"No serviço de saúde A tem a vantagem de ter lixeiras rotuladas com classificação e os símbolos dos resíduos, tipo preto para comum, branco para contaminado" (D1).

Nos depoimentos ainda pode-se inferir que a escassez de recursos materiais dificulta o processo de ensino-aprendizagem. Segundo Brit, Rozendo e Melo (2018), o laboratório de habilidades quanto mais se aproximar da realidade, mais crítico se torna e mais propenso a contribuir para a formação de enfermeiros capazes de intervir na realidade com vistas à sua transformação. Estes espaços demandam recursos humanos e materiais para o atendimento às necessidades de assistência e aprendizagem.

\footnotetext{
"No laboratório não tem lixo para ser jogado, é tudo muito faz de conta, você tem o saquinho, mas não tem realmente o que jogar, tipo você tem uma gaze invisível e despreza no saquinho. Na prova a gente esqueceu de levar o saquinho e todo mundo perdeu ponto" (D5).
}

"Como a colega falou, no laboratório a gente tem problemas como a falta de material por exemplo: gaze, luvas geralmente estão colabadas” (D9).

Uma pesquisa que teve como objetivo verificar o conhecimento sobre o descarte de resíduos infectantes e a prática entre acadêmicos dos cursos de Enfermagem e de Medicina de uma universidade pública evidenciaram que o conhecimento dos discentes sobre descarte de resíduos infectantes mostrou-se frágil e insuficiente para garantir a sua aplicabilidade na prática (Souza et al., 2015).

É necessário investimento em um ambiente de formação que propicie reflexão, problematização, crítica, articulação e comprometimento com a construção de sujeitos que incorporem posturas éticas, de solidariedade, de consciência cidadã, de compromisso social, atuando de forma responsável para com o meio ambiente (Corrêa, Lunardi \& Santos, 2008).

Vale ressaltar também que o manejo inadequado dos resíduos de serviços de saúde contribuem para uma reflexão ampliada e crítica da prática em saúde, de modo que é necessária a compreensão dos discentes que tal ação negativa não se limita apenas a nível local, mas de modo mais amplo, a nível global (Guimarães et al, 2021). 
Nesse sentido, acredita-se que essas atividades de ação-reflexão, resultando em ação com transformação, possam contribuir para a responsabilização nas questões que dizem respeito aos RSS, sobretudo na dimensão de agressão à saúde pública. Para tanto, é importante que a temática sobre RSS, na graduação, aconteça em vários momentos do curso, com graus crescentes de complexidade, e tenha como grande pilar a biossegurança.

\section{Considerações Finais}

O estudo constatou que existem fragilidades no processo de formação dos enfermeiros frente à temática RSS. Possivelmente, esse fato esteja associado à fase do curso vivenciada pelos discentes pesquisados. Destarte, esses resultados merecem ser investigados em estudos futuros, para aprofundamento, buscando soluções para melhorar o ensino sobre o tema. Não obstante, pode-se considerar que os resultados encontrados nessa pesquisa não se apresentaram divergentes aos diversos estudos dos últimos cinco anos, os quais demonstram uma abordagem teórica superficial e com experiências práticas escassas.

Entre as recomendações desse estudo, visando ao aprimoramento do conhecimento dos discentes sobre o tema e tornando o egresso mais capacitado, destacam-se: o fomento ao aprendizado por meio de metodologias de ensino inovadoras, contextualizadas, articuladas e problematizadas, que sejam capazes de instrumentalizar os discentes para o enfrentamento dos RSS de saúde de forma responsável e consciente; a inclusão do tema de forma transversal na matriz curricular do curso; a realização de seminários temáticos, debates, fórum de discussão, cursos presenciais, cursos a distância, pesquisas e publicações sobre o tema, entre outros. Por fim, são importantes a manutenção e a criação de novos espaços de reflexão sobre o tema, em consonância com as políticas e os pressupostos teóricos, tendo como grande pilar a biossegurança global.

Portanto, novos estudos são necessários para maior aprofundamento do assunto abordado, envolvendo não só o conhecimento de estudantes de enfermagem durante a graduação, como também de enfermeiros graduados. Portanto, espera-se que este artigo possa contribuir com pesquisas futuras.

\section{Referências}

André, S., Veiga T. \& Takayanagui A. (2016). Geração de Resíduos de Serviços de Saúde em hospitais do município de Ribeirão Preto (SP), Brasil. Engenharia Sanitária e Ambiental, 21(1), 123-130.

Bento D. et al. (2017). O gerenciamento de resíduos de serviço de saúde sob a ótica dos profissionais de enfermagem. Texto Contexto - Enfermagem, 26 (1), e6680015.

Brasil. Ministério da Educação. Conselho Nacional de Educação. Câmara de Educação Superior. Resolução CNE/CES nº 3, de 7 de novembro de 2001. Institui Diretrizes Curriculares Nacionais do Curso de Graduação em Enfermagem. Brasília, DF: MEC, 2001. http://portal.mec.gov.br/cne/arquivos/pdf/CES03.pdf

Brasil. Ministério da Saúde. Agência Nacional de Vigilância Sanitária. Resolução RDC n 222, de 28 de março de 2018 . Regulamenta as Boas Práticas de Gerenciamento dos Resíduos de Serviços de Saúde e dá outras providências. Brasília, DF: ANVISA, 2018. http://portal.anvisa.gov.br/documents/10181/3427425/RDC_222_2018_.pdf/c5d3081d-b331-4626-8448-c9aa426ec410.

Brito, F., Rozendo, C. \& Melo, P. (2018). Laboratório de enfermagem e a formação crítica de enfermeiros: aproximações e distanciamentos. Revista Brasileira de Enfermagem, 71(4), 1589-96.

Cofen. Resolução 303, de 23 de junho de 2005. Dispõe sobre a autorização para o enfermeiro assumir a coordenação como responsável técnico do plano de gerenciamento de resíduos de serviços de saúde. Rio de Janeiro: COFEN, 2005. http://www.cofen.gov.br/resoluo-cofen-3032005_4338.html

Corrêa, L., Lunardi, V. \& Conto, S. (2007). O processo de formação em saúde: o saber resíduos sólidos de serviços de saúde em vivência práticas. Revista Brasileira de Enfermagem, 60 (1), 21-25.

Corrêa, L., Lunardi, V. \& Santos, S (2008). Construção do saber sobre resíduos sólidos de serviços de saúde na formação em saúde. Revista Gaúcha de Enfermagem, 29(4), 557-564.

Eduardo, A. et al. (2016). Scenario for a simulation of health services' waste: a methodological study. Online Brazilian Journal of Nursing, 15 (4), 611-616.

Garbin, A. et al. (2015). A visão dos acadêmicos de odontologia sobre o gerenciamento dos resíduos do serviço de saúde. Archives of Health Investigation, 4(4), 63-67.

Gomes A. et al. (2017). Sustentabilidade ambiental: gerenciamento de resíduos odontológicos no Serviço Público. Revista Cubana de Estomatologia, 54 (2), 1 11. 
Research, Society and Development, v. 10, n. 2, e16310212280, 2021

(CC BY 4.0) | ISSN 2525-3409 | DOI: http://dx.doi.org/10.33448/rsd-v10i2.12280

Gomes, C. et al. (2014). Biossegurança e resíduos de serviços de saúde no cotidiano acadêmico. Revista de Ciências Farmacêuticas Básica e Aplicada, 35 (3), 443-450.

Guimarães, P. et al. (2021). Percepção discente acerca das consequências do descarte inadequado dos resíduos de saúde. Research, Society and Development, $10(1)$, e43610111915, 1-7.

Moreschi, C. et al (2014). A importância dos resíduos de serviços de saúde para docentes, discentes e egressos da área da saúde. Revista Gaúcha de Enfermagem. $35(2), 20-26$

Moreschi, C., Rempel C. \& Backes D. (2014). A percepção de docentes de cursos de graduação da área da saúde acerca dos resídu os de serviços de saúde. Revista Baiana de Saúde Pública, 38 (3), 647-664.

Oliveira, L. et al. (2018). Fatores associados ao manejo adequado de resíduos de serviços de saúde entre profissionais de enfermagem. Revista Baiana de Enfermagem, 32 (e25104).

Pereira, A., Shitsuka, D, Parreira, F. \& Shitsuka, R. (2018). Metodologia da pesquisa científica. Ed. UAB/NTE/UFSM. https://repositorio.ufsm. br/bitstream/handle/1/15824/Lic_Computacao_MetodologiaPesquisa-Cientifica.pdf.

Peres C., Andrade, A. \& Garcia, S. (2007). Atividades Extracurriculares: Multiplicidade e Diferenciação Necessárias ao Currículo. Revista Brasileira de Educação Médica, 31(3), 203-211.

Sanchez, A. et al. (2018). Resíduos de Serviços de Saúde: conhecimento de enfermeiros da Atenção Básica. Revista Brasileira de Enfermagem, 71 (5), 2508 2517.

Santos, M. et al. (2019). Degree in Nursing: education through problem-based learning. Revista Brasileira de Enfermagem, 72(4), 1071-1077.

Souza, A. et al. (2015). Descarte de resíduos infectantes: informações demonstradas e ações praticadas por estudantes de enfermagem e medicina. Revista Eletrônica de Enfermagem, 17 (1), 124-130.

Uehara, S., Veiga, T. \& Takayanagui, A. (2019). Gerenciamento de resíduos de serviços de saúde em hospitais de Ribeirão Preto (SP), Brasil. Engenharia Sanitária e Ambiental, 24(1), 121-130.

Zarpelão, R. et al (2018). Psychosocial Factors and Their Relation Between Work Accidents Among Outsourced Construction Workers. International Journal of Precious Engineering Research and Applications (IJPERA), 3 (1), 67-71. 